\title{
CYBERSECURITY HEALTH CHECK
}

A Framework to Enhance Organizational Security

\section{SHIH MING PAN, CHII-WEN WU, PEI-TE CHEN, YUN TING LO, AND PEI WEN LIU}

\section{Contents}

Introduction 276

Theoretical Basis of Cybersecurity Health Check (CHC) 277

Strategy Map 277

Balanced Scorecard (BSC) 280

Definitions of Items 280

Hierarchical Structure of Measurement Metrics 283

Detailed Infrastructure in CHC 285

Security Awareness and Education Perspective 285

ISMS Establishment Perspective 286

Defense-in-Depth Implementation Perspective 287

Protection Requisitions Perspective 288

The CHC Execution Process 288

Phase 1: Preparative Operation of Health Check 288

Phase 2: Information Security (IS) Protection Deployment

Testing 289

Phase 3: IS Management System Implementation Review 289

Phase 4: CHC Analysis and Report 290

Conclusion 291

References $\quad 292$ 


\section{Introduction}

Organizations around the world are increasingly using information technology (IT) and the Internet to offer better service to their constituents. IT is now considered a critical asset, and most organizations have implemented some form of security protection in response to cyber threats. In order to manage this risk properly, particular attention is paid to information security (IS) management, such as raising the awareness of employees, improving the skills of IT staff, introducing Information Security Management Systems (ISMSs), or applying security equipment, such as firewalls, intrusion-detection systems (IDSs), or intrusion prevention systems (IPSs) to ensure network security. All these preventive measures increase the information security budget; however, the real question is whether money is being spent on the necessary preventive measures and equipment, and whether they are really effective in protecting organizations' IT assets.

In fact, a lot of security threats are hidden in an organization due to user errors, the ineffectiveness of IS systems, or unknown external malicious acts. In order to lower these risks, many organizations have introduced ISMS based on the ISO/IEC 27001 standard, established in 2005 by the International Organization for Standardization (ISO) and the International Electrotechnical Commission (IEC). This standard provides specifications for bringing information security under management control and allows organizations to implement security control measures according to the standard's defined security policy and operational procedures, which can then be audited and certified accordingly.

In many cases, organizations would assume that they are able to identify and solve information security problems by introducing ISMS. But ISMS has two major problems. First, only security issues within its scope are audited, which means that auditors simply examine whether a policy is implemented or not; however, security issues outside of the scope are not considered. Second, ISMS results provided by auditors only provide nonconformances, not quantifiable data. Management maestro Peter Drucker once said that "what cannot be measured cannot be managed." If an organization wants to take risk control measures to reduce information security risk, it has to properly quantify information security risk and minimize the preventable risk, and the cost of loss. This is why the ISO is currently 
developing ISO 27004, Metrics and Measurement of Information Security Management, as announced on December 7, 2009.

To improve the problems of ISMS, the Institute for Information Industry (III), a nongovernmental organization that supports the development and applications of the information industry as well as the information society in Taiwan, set up the Information and Communication Security Technology Service Center (ICST), a taskforce. The mission for ICST is to assist Research, Development, and Evaluation Commission (RDEC) of Executive Yuan (the Taiwanese Cabinet), to provide an early security warning in advance, and to recover systems after the forensic and other technical services. In this chapter, ICST introduces the Cybersecurity Health Check (CHC) framework to measure the performance of an organization's information security protection with a set of quantifiable metrics.

Because of the current lack of systematic measurement of IS performance, $\mathrm{CHC}$ builds on two business strategies: the theoretical perspectives of Strategy Map and Balanced Scorecard (BSC). This helps to improve security through internal and external protective mechanisms and on-site measurement. The $\mathrm{CHC}$ framework can assist organizations to identify their potential security threats and adopt needed protection solutions in advance. This will allow organizations to strengthen their security levels and lower the cost of loss.

\section{Theoretical Basis of Cybersecurity Health Check (CHC)}

The CHC framework measures the performance of an organization's information security protection with a set of quantifiable metrics. These metrics are created based on the management theories of Strategy Map and Balanced Scorecard (BSC) as follows.

\section{Strategy Map}

Strategy Map is a strategic planning tool proposed by Professors Robert S. Kaplan and David P. Norton (1996, 2006) of Harvard University in 1990. It includes two components: "Strategy" and "Map." Strategy is the action plan for specific objectives, and Map is the graphical representation of the strategy. That is, "Strategy Map" can be described as "an action plan road map to achieve a specific value proposition." On 


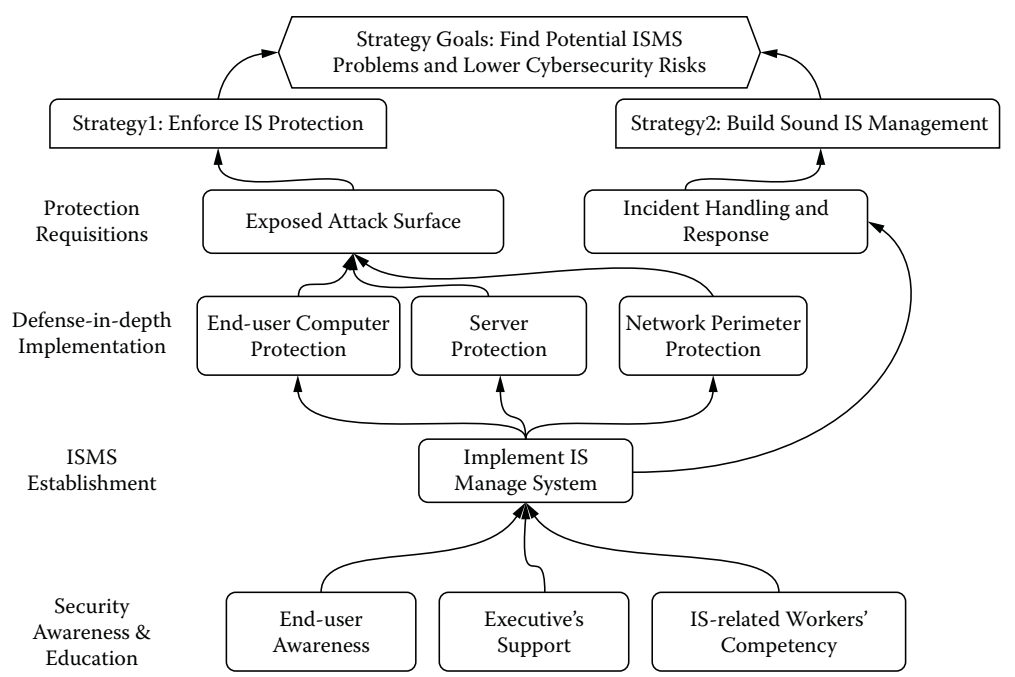

Figure 11.1 Cybersecurity Strategy Map.

the other hand, Strategy Map should clearly explain the causal and logical relationship of various strategies.

When applied to information security, the concept of Strategy Map is to emphasize the integrated resources of IS management and protection deployment. We assume the strategic goal of cybersecurity is to "find potential ISMS problems" and "lower cybersecurity risks" in an organization. In order to achieve this strategic goal, we have to find the road map and causal relationship of management and technology first, and then produce the Strategy Map of cybersecurity, as shown in Figure 11.1.

According to the casual relationship and theory of Strategy Map in Figure 11.1, four perspectives are used to reduce the risk of information security incidents, each of which will be discussed in turn: Security Awareness and Training, ISMS Establishment, Defense-inDepth Implementation, and Protection Requisitions.

Security Awareness and Training/Education People are the most critical factor in cybersecurity protection, but also the weakest link in the system. If an organization wants to achieve the strategic goal of reducing security risks, it should provide IT users with training. In doing so, end-users will recognize the importance of security protection to their jobs and be willing to follow the policies and regulations accordingly. 
Another critical factor is whether staff members responsible for cybersecurity within the organization have enough awareness and technical competency to protect the organization from external attacks. If the IT staff members do not have the fundamental knowledge or expertise, the organization could be paralyzed when encountering security threats and make the situation worse. Last, the delegation and support of top management is also a key factor in determining the success of an action plan. After completing the fundamental training of its personnel, an organization can enact an information security policy and management system to ensure the safety of its information equipment and data.

Information Security Management System (ISMS) Establishment Considering resource limitations, an organization may base its IS management policies and procedures on ISMS to protect its IT assets. When developing ISMS, an organization has to make sure that the policy and control measures are implemented. If there are no security policies, employees have nothing to follow. If the organization has established security policies but does not implement them correctly and effectively, then ISMS will become a mere formality. It is recommended that the PDCA (Plan, Do, Check, and Act) model proposed by W. Edwards Deming could be used to examine effectiveness of ISMS at an organization.

Defense-in-Depth Implementation Once ISMS is in place, the deployment of protection equipment is needed to meet the organization's requirement in IS management criteria and control measures. The organization should focus on risk assessment. In addition, it should also keep track of the implementation of the equipment to prevent against internal or external information threats.

Protection Requisitions After tackling the above three perspectives, it does not mean an organization can just sit back and relax due to the rapid development of security attacks. In addition to well-known security issues, organizations should actively guard against unknown threats through security drills, incident reporting and response mechanisms, and so on. Organizations should continue to monitor 
all ISMS measures and maintain solutions for the latest information security information, and take necessary preventive measures.

\section{Balanced Scorecard (BSC)}

Balanced scorecard (BSC) is a business strategic management tool, also developed by Kaplan and Norton $(1996,2006)$. The concept of BSC focuses on finding the balance between the short-term and longterm goals, financial and nonfinancial measurements, lagging and leading indicators, as well as internal and external performances.

BSC can be regarded as a quantifiable indicator for organizations to review their performance on various fronts. Traditionally, a BSC system reviews the performance from four perspectives: financial, customer, internal, and learning and growth. Managers can determine different perspectives and performance measurement metrics based on different targets and strategies and match them with organizational strategy and vision to help each department to reach their objectives. BSC enables an organization to achieve its goal or mission by aligning action and vision; therefore, it can also be regarded as a performance measurement system that describes, communicates, and executes strategies.

$\mathrm{BSC}$ is also used to convert organizational vision and strategy into objectives and measurements. It is intended not only to develop measureable items, but to achieve the goals of management and execution in a "balanced" way. Therefore, this study designed a cybersecurity BSC (as shown in Figure 11.2) by referencing the IS protection experience. It tried to identify the vulnerabilities that exist between the ISMS strategy and IS protective operations by the indicators from different IS perspectives. It also tried to identify the performance from the key performance indicators (KPIs) and driving factors.

\section{Definitions of Items}

$\mathrm{CHC}$ often uses perspectives, critical activities, KPIs, and driving factors to describe the whole framework and also uses them to deduce the weak link in organizations. This section provides an overview of this terminology. 


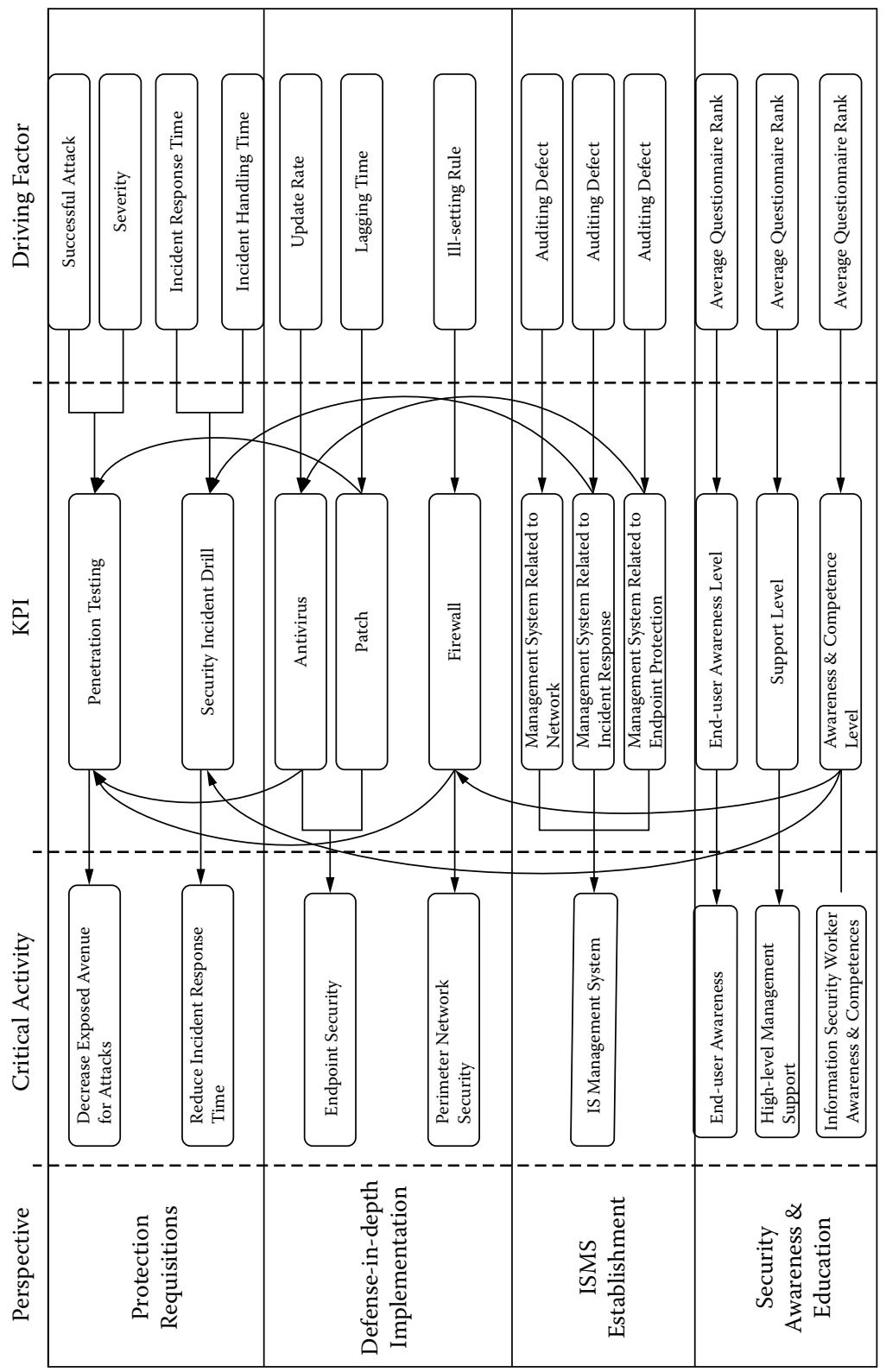

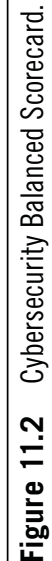


Perspectives: Perspectives refers to the different dimensions of IS protection in organizations. Once the performance differences in various perspectives are too large, it means the organization is in the situation of uneven distribution of security resources. At this time, the organization needs to review the allocation of security resources in order to avoid too much emphasis on one perspective and cause security problems in another.

In CHC, there are four perspectives: protection requisitions, defense-in-depth implementation, ISMS establishment, and security awareness and education/training.

Critical Activities: Critical activities are possible causes of perspectives and are the important factors affecting each perspective. They are often used to view the performance of organizational security operations. When these operations are not implemented properly, the organization's critical activities will be in a lower class. If not detected, or to deal with these critical activities, the organization may face potential information security vulnerabilities and problems.

Key Performance Indicators (KPIs): Key Performance Indicators (KPIs) are significant indicators of cause in each critical activity. The KPIs defined in CHC have to be effective, efficient, and matched with the strategic goals. The indicators also have to be consistent with the principles of SMART, a way of setting targets or evaluating objectives, as follows:

(1) Specific: the measurement items and results have to be specific to ensure consistent interpretation and the feasibility of measurement.

(2) Measureable: the performance indicators will be used for comparison purpose on a yearly basis for the organization; therefore, the results need to be measurable so that the organizations would know what and how to improve.

(3) Attainable: the consideration of the complexity of business activities, labor and financial cost concerns, and time efficiency. The performance indicators should aim at a higher but attainable performance standard.

(4) Relevant: the performance indicators should be determined according to the proper categories and implementation data. 
(5) Time-bound: measurement of the performance indicator should be time-bound so that people responsible for $\mathrm{CHC}$ would know when it can be achieved. The criteria can also be referred to as "timeliness."

Driving Factors: Driving factors are the executed "result" of present protection measures in organizations. It is the detailed contents of KPIs, reflecting the status of the most basic of all perspectives. The current performances of driving factors affect the scope and impact of potential security problems. Organizations can recognize the recent protective measures implementation through driving factors and locate security protection measures to improve the needed driving factors. This way, possible security incidents can be avoided.

\section{Hierarchical Structure of Measurement Metrics}

The metrics of $\mathrm{CHC}$ can be divided into six levels: overview, perspectives, critical activity, KPI, driving factor, and raw data. Each level will affect the performance of the follow-up indicators, as illustrated in Figure 11.3.

The CHC framework uses statistical analysis methods, such as the Likert scale, a measurement methodology created by Rensis Likert, which is mainly applied in the fields of social science and psychology. According to different research purposes, the Likert scale can be divided into five or seven different scales, of which each is given a specific definition. As well as actual recording or

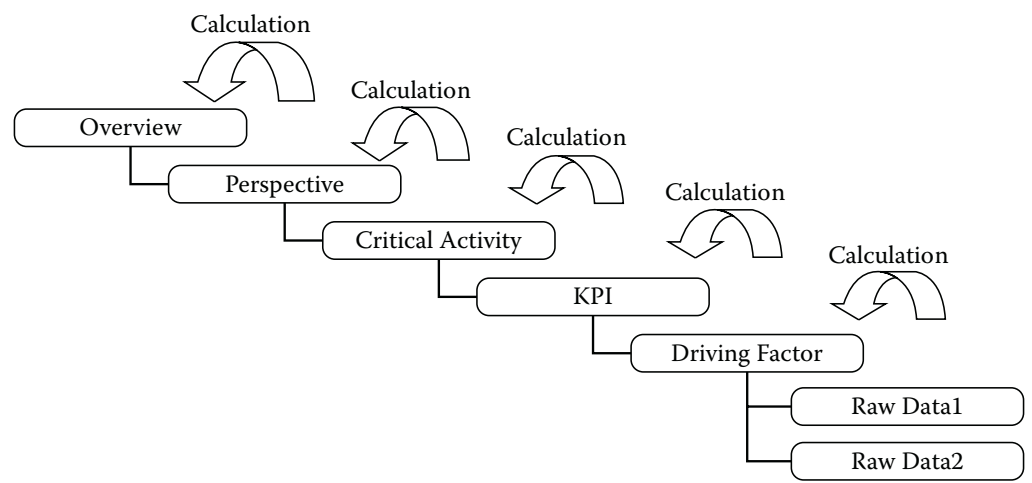

Figure 11.3 Measurement Metrics Hierarchy. 
Incident Handling and Response (Critical Activity) = Security Alert Response (KPI) + Incident Handling (KPI)

\begin{tabular}{|c|c|c|c|}
\hline $\begin{array}{c}\text { Security Alert } \\
\text { Response Interval (R) }\end{array}$ & Rank (R) & $\begin{array}{l}\text { Incident Handling } \\
\text { Interval }(\mathrm{H})\end{array}$ & $\operatorname{Rank}(\mathrm{H})$ \\
\hline No alerts & 11 & No alerts & 11 \\
\hline$(0,1]$ & 10 & $(0,1]$ & 10 \\
\hline$(1,2]$ & 9 & $(1,2]$ & 9 \\
\hline$(2,3]$ & 8 & $(2,3]$ & 8 \\
\hline$(3,4]$ & 7 & $(3,4]$ & 7 \\
\hline$(4,5]$ & 6 & $(4,5]$ & 6 \\
\hline$(5,6]$ & 5 & $(5,6]$ & 5 \\
\hline$(6,7]$ & 4 & $(6,7]$ & 4 \\
\hline$(7,8]$ & 3 & $(7,8]$ & 3 \\
\hline$(8,9]$ & 2 & $(8,9]$ & 2 \\
\hline$>9$ & 1 & $>9$ & 1 \\
\hline & $\begin{array}{l}\text { Incident Handling } \\
\text { and Response }\end{array}$ & Rank & \\
\hline & $>20$ & 11 & \\
\hline & $19 \sim 20$ & 10 & \\
\hline & $17 \sim 18$ & 9 & \\
\hline & $15 \sim 16$ & 8 & \\
\hline & $13 \sim 14$ & 7 & \\
\hline & $11 \sim 12$ & 6 & \\
\hline & $9 \sim 10$ & 5 & \\
\hline & $7 \sim 8$ & 4 & \\
\hline & $5 \sim 6$ & 3 & \\
\hline & $3 \sim 4$ & 2 & \\
\hline & $<3$ & 1 & \\
\hline
\end{tabular}

Figure 11.4 Key performance indicator (KPI) normalization.

data analysis, questionnaires can also be used to acquire scale values based on different responses and survey subjects to review each perspective in the IS field. The Likert Scale is also used for the data conversion into 11 levels in $\mathrm{CHC}$ to normalize the evaluation results, as shown in Figure 11.4. Without the process of normalization, the values of each indicator would vary too much to be evaluated in a consistent format.

All analysis processes in the $\mathrm{CHC}$ framework are designed according to a hierarchy. In the first place, we select a group of driving factors from the raw data by using correlation analysis, principal component analysis, factor analysis, and so forth. Then we conduct various cross-analyses to come up with the result, which are the KPIs in our 
framework. Layer by layer, we delve further into detail to find the critical activities and perspectives.

The values of average and standard deviation are calculated by the principle of normal distribution as the benchmark for measurement. If the measurement result is higher than the benchmark value, we may consider the IS protection performance in that specific area above average. Equally, if the measurement result is lower than the benchmark, it means that the organization's IS protection performance is below average and therefore needs to be reviewed and improved. In the CHC framework, qualitative and quantitative analyses are adopted to probe possible impacts and effects.

\section{Detailed Infrastructure in $\mathrm{CHC}$}

As mentioned earlier, the four perspectives in Strategy Map are used to help organizations learn the status quo of IS protection, evaluate the protection performance, and identify possible security threats and vulnerabilities to make amendments and lower the probability of security incidents. In this section, we return to each perspective of Strategy Map and describe its detailed structure, including the characterization of driving factors, KPIs, and critical activities.

\section{Security Awareness and Education Perspective}

Figure 11.5 illustrates security awareness and training. This perspective includes three critical activities: "user awareness," "IS-related worker competency," and "executive support." Under the CHC framework, personal interviews and awareness surveys will be carried out on general users, security personnel, and chief information security officer (CISO) to see whether internal staff has basic security awareness, or the capability to deploy protection equipment for the organization. The perspective can also verify whether the internal staff of an organization is familiar with information security policy, management process, and operational procedures to evaluate the performance of an IS management system. 


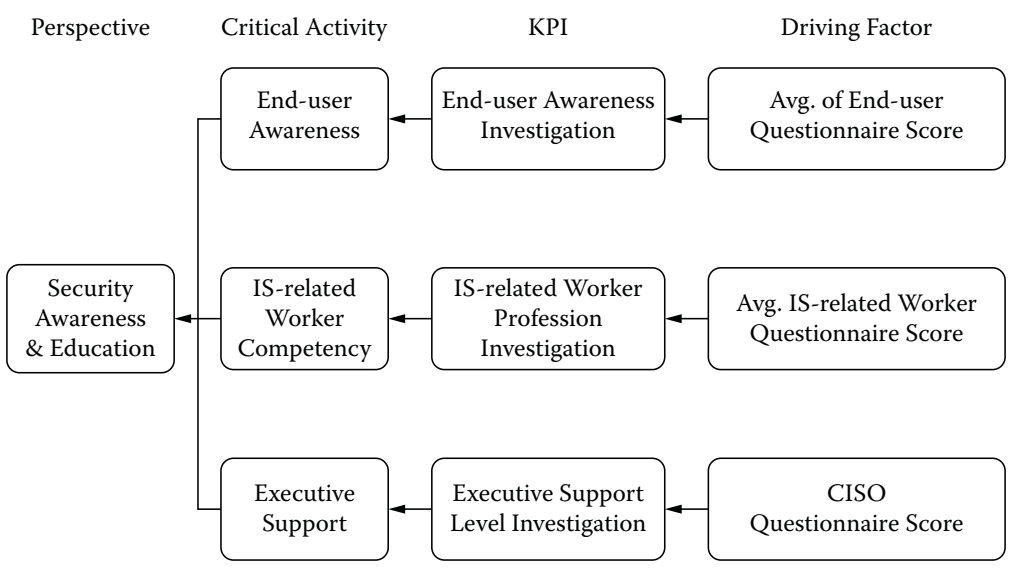

Figure 11.5 Security awareness and education structure.

\section{ISMS Establishment Perspective}

As shown in Figure 11.6, the scope of this perspective goes beyond the certification of ISO/IEC 27001 as it inspects every security management policy, process, and procedure based on the security issues identified. The purpose of $\mathrm{CHC}$ is not to help the organization to achieve a security certification, but to help the organization identify the vulnerabilities of IS systems in order to take preventive measures and correct issues. Further, because not all departments and

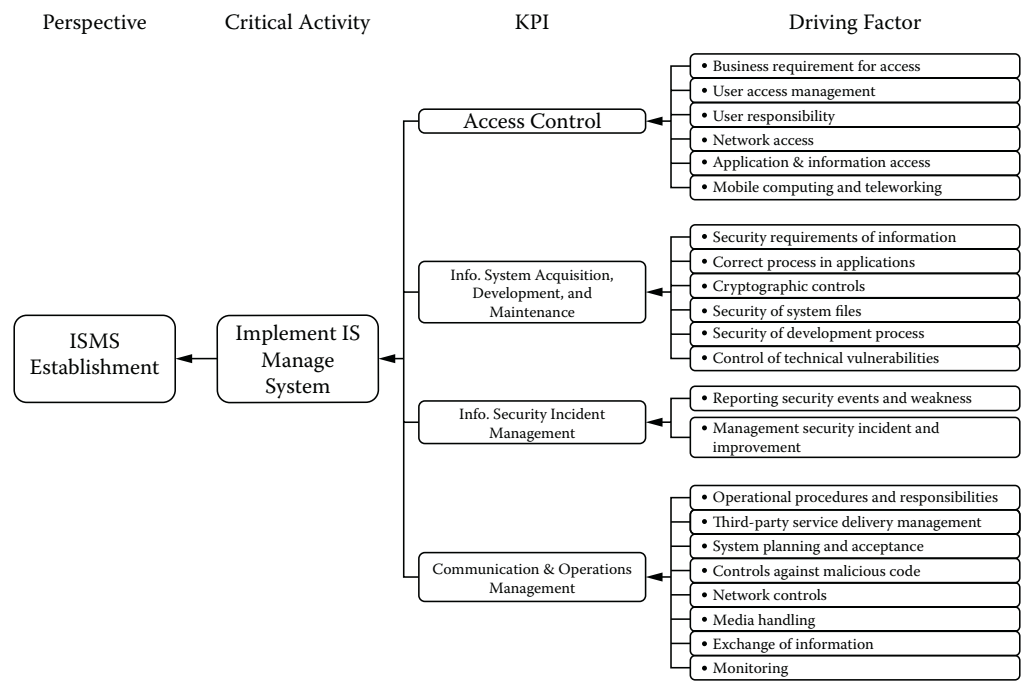

Figure 11.6 Information Security Management System (ISMS) establishment structure. 
information systems are included in the scope of ISMS certification, it is essential to know how an organization would respond to security incidents that are outside of the scope of information security management certification.

The implementation of this perspective focuses on the issues of access control; IS acquisition, development, and maintenance; IS incident management; and communication and operations management. It is intended to ensure proper implementation of ISMS and the effectiveness of IS protection measures.

\section{Defense-in-Depth Implementation Perspective}

As shown in Figure 11.7, this perspective includes three critical activities: end-user computer protection, server protection, and network perimeter protection. It is implemented by conducting a host examination on user's computers and servers. End-user computer and server protection examination KPIs include the coverage and update of antivirus software, security level of user password configuration, potential risk of computer configuration, and whether malicious programs have existed in user's computer and server. KPIs of network border security include whether the firewall can filter or block malicious packets. The network architecture of the organization will also be examined to identify vulnerabilities.

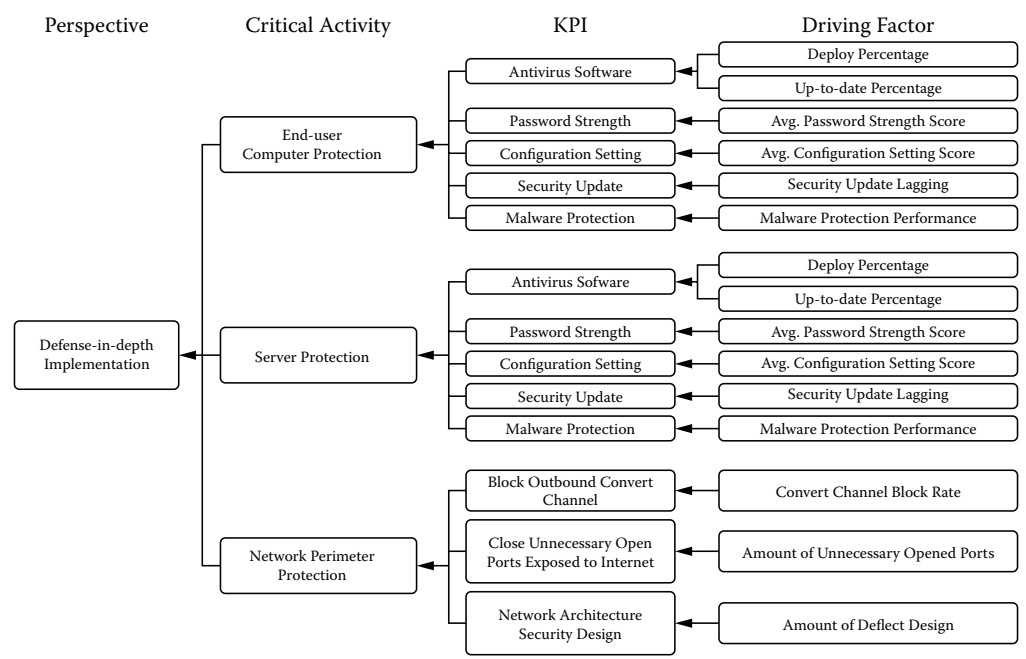

Figure 11.7 Defense-in-depth implementation structure. 


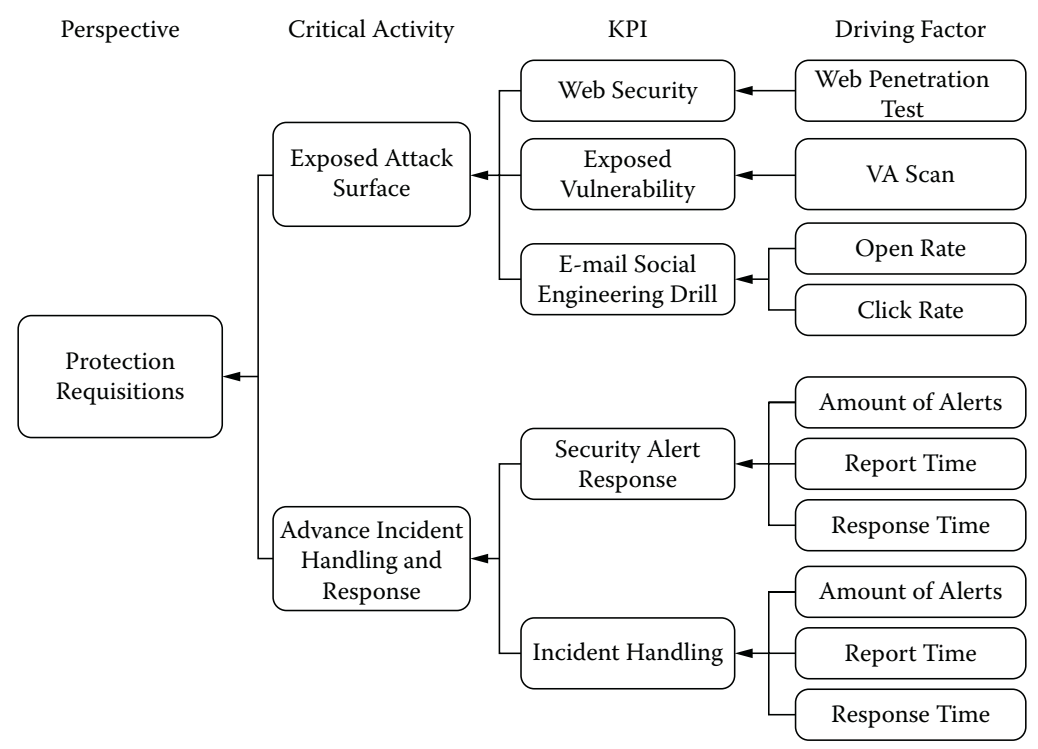

Figure 11.8 Protection requisitions structure.

\section{Protection Requisitions Perspective}

This perspective (shown in Figure 11.8) includes two critical activities: exposed attack surface and advance incident handling and response. It is implemented by evaluating the organization's website service security, external-service host vulnerability scanning, awareness of social engineering e-mail, and the organization's reporting/response on IS incidents. It is intended to evaluate whether an organization has sufficient protection and response capability when encountering security threats and identify the blind spots and vulnerabilities to minimize security incidents.

\section{The CHC Execution Process}

When an organization wants to identify its security level, the CHC execution process includes four phases, as follows: Preparative Operation of Health Check, IS Protection Deployment Testing, IS Management System Implementation Review, and CHC Analysis and Report.

\section{Phase 1: Preparative Operation of Health Check}

The first phase provides $\mathrm{CHC}$ questionnaires to the responsible personnel to fill out. This questionnaire is intended to capture the 
existing ISMS, protection deployment, and the scope of $\mathrm{CHC}$ for the reference of service scope definition and sampling. In addition, the responsible personnel should organize the implementation of $\mathrm{CHC}$ with the schedule and the necessary items, so CHC operators can perform the check operations smoothly. The output of this phase is an execution plan, including the basic information survey and execution schedule.

\section{Phase 2: Information Security (IS) Protection Deployment Testing}

$\mathrm{CHC}$ operators will conduct technical measurement and testing with automated tools in the second phase. These tools collect information, such as configuration, update status, antivirus status and password strength, and so forth, from a PC or server, in order to examine the end-user computer protection, server protection, and network perimeter protection. Other information on $\mathrm{PC}$ s or servers that cannot be gathered automatically by tools, such as malware protection, network architecture design, will be examined manually on-site by $\mathrm{CHC}$ operators. In addition, end-users and IT department staff will be randomly selected to fill out questionnaires designed to recognize their security awareness and education.

Moreover, two critical activities, "Exposed attack surface" and "Advance incident handling and response" are performed remotely. To examine the organization's exposed attack surface, CHC operators will do a penetration test on the organization's website and send out social engineering e-mails to employees to see the open and click rates. To check the organization's incident handling and response, $\mathrm{CHC}$ operators select security alert response and incident handling records from ICST database to count the amount of alerts, report time, and response time.

The output of this phase is the necessary raw data for three perspectives, Security Awareness and Education, Defense-in-Depth Implementation, and Protection Requisitions.

\section{Phase 3: IS Management System Implementation Review}

In the third phase, $\mathrm{CHC}$ operators will interview the ISMS managers or IT staffs based on the indicators identified in the perspectives 
of Protection Requisitions, Defense-in Depth Implementation, and Security Awareness and Training. The result can help CHC operators understand whether the organization has established the relevant or appropriate control measures, and determine whether an appropriate implementation of ISMS is in place based on the technical test results. By reviewing the organization's ISMS implementation, such as access control, security incident management, and communication management, $\mathrm{CHC}$ operators can identify deficiencies in an organization and make recommendations for improvement and preventive measures. Thus, the output of this phase are the recommendations for the ISMS Establishment perspective.

\section{Phase 4: CHC Analysis and Report}

In order to determine the protection performance and identify potential vulnerabilities, all the collected raw data will be normalized and calculated by the hierarchical structure, described earlier. The calculated results are then presented in a radar chart, as exemplified by Figure 11.9 that shows an example of a $\mathrm{CHC}$ analysis. As we can see most of the KPI scores are relatively high, which means the organization's security protection are above average. However, we also observe three KPI scores are relatively poor: end-user awareness, e-mail social engineering drill, and malware protection. We may conclude that in this case, the end-users may open social engineering e-mails designed by hackers because of a lack of security awareness, resulting in users' computers being infected by malicious software. Therefore, we would recommend to the organization that it should enhance end-users' security awareness education and training, thereby reducing potential security threats.

According to the calculated results, we will generate the $\mathrm{CHC}$ Summary Report. This report describes the relevance between each item in the radar chart and shows organizations' security performance and the most vulnerable point in the current security environment. This provides organizational CISOs with the reference they need to improve protection deployment. All the $\mathrm{CHC}$ data are stored in a database as a reference to see whether the organization has made corrections and improved over time. 


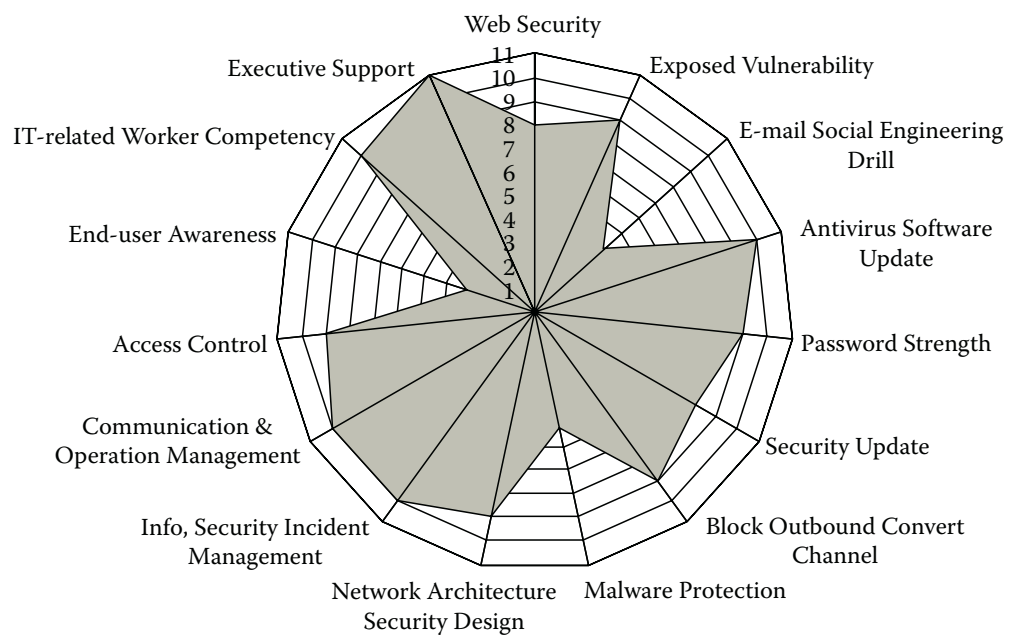

Figure 11.9 A radar chart example of Cybersecurity Health Check (CHC).

\section{Conclusion}

In this chapter, we presented the $\mathrm{CHC}$ framework that is based on the business concepts of Strategy Map and BSC. The framework helps to improve organizational security protection through internal and external protective mechanisms and on-site measurement. With $\mathrm{CHC}$, organizations can identify their potential security threats and adopt protection solutions in advance, which in turn can strengthen their security level and lower costs.

ICST is currently using this framework in more than 10 government authorities in Taiwan. Results thus far show that in general, government agencies achieve a higher level of security in the Security Awareness and Education and ISMS Establishment perspectives due to efforts in promoting ISO/IEC 27001. Meanwhile, agencies perform less well in the Defense-in-Depth Implementation and Protection Requisitions perspectives. This shows that government agencies do not appropriately implement their defined ISMS. Specifically, we observed potential threats in three critical activities, "End-user Computer protection," "Server protection," and "Exposed attack surface." The most fundamental reason comes from the malware protection and web security KPIs. Therefore, we would recommend for these government agencies to strengthen the effectiveness of protection, such as sampling tests of the user's computer and server 
security, and regular vulnerability scanning or penetration testing. With CHC, we can find the information security of government agencies in the vulnerabilities and make recommendations for improvement to make the decision makers, such as CISOs, understand how information security budgets must be used effectively.

The CHC framework was designed for public sector agencies in Taiwan but can be applied broadly, to government agencies around the world or to private sector entities. Anyone who wants to examine security with this framework can define his or her own ranking values, derive the most vulnerable part of the environment with the defined driving factors, KPIs, Critical Activities, and Perspective based on Strategy Map and BSC, and make the most efficient use of budget.

\section{References}

Corporate Information Security Working Group. Report of the Best Practices and Metrics Teams, Subcommittee on Technology and Information Policy, Intergovernmental Relations and the Census, Government Reform Committee, U.S. House of Representatives (Rev. January 10, 2005).

Jaquith, A. Security Metrics: Replacing Fear, Uncertainty, and Doubt, Boston: Addison Wesley Professional. 2007.

Kaplan, R. S., and Norton, D. P., Strategy Maps: Converting Intangible Assets into Tangible Outcomes. Boston: Harvard Business School Publishing, 2004.

Kaplan, R. S., and Norton, D. P. Alignment: Using the Balanced Scorecard to Create Corporate Synergies. Boston: Harvard Business School Press, 2006. Kaplan, R. S., and Norton, D. P. The Balanced Scoreboard: Translating Strategy into Action. Boston: Harvard Business School Publishing, 1996.

Kaplan, R. S., and Norton, D. P. The Strategy-Focused Organization: How Balanced Scorecard Companies Thrive in the Nerw Business Environment. Boston: Harvard Business School Press, 2000.

Swanson, M., Bartol, N., Sabato, J., Hash, J., and Graffo, L. Security Metrics Guide for Information Technology Systems, NIST Special Publication 80055 (Washington, DC: National Institutes of Standards and Technology, 2003). 